\title{
An In-field Calibrated Multiband-Multiresolution Bilateral Filter for Digital Image Applications
}

\author{
Fabiana O. P. Fernandes, Flávia M. F. Ferreira, Member, IEEE, Zélia M. A. Peixoto, Member, IEEE \\ and Andrei S. Silva
}

\begin{abstract}
This work presents the multi-band multi-resolution bilateral filter (MMBF) as a novel approach to the multiresolution bilateral filter (MBF), which associates the bilateral filter with wavelet denoising techniques. In addition to embedding 2D-BFs in different wavelet resolutions, it also introduces onedimensional bilateral filters (1D-BFs) in the sub-bands corresponding to the horizontal and vertical details. Developments regarding an in-field calibration method for the MMBF are also reported in order to assess its full effectiveness. The noise levels are reduced concomitantly with the preservation of edges and details, meeting the requirements of image quality. To validate the proposed method, we present a case study based on phantoms imaged by a piece of medical X-ray equipment. These images undergo severe degeneration when obtained under conditions of low radiation dosage. After both have been calibrated using the method proposed, the ability of MMBF to preserve image details while reducing noise surpasses the MBF by quantitative assessment as well as by visual inspection.
\end{abstract}

Index Terms-Image Denoising, Image Filtering, Measurement Calibration, Multiband-Multiresolution Bilateral Filter, X-ray Detection.

\section{INTRODUCTION}

I MAGE denoising is an essential area of research in various fields of image processing, such as segmentation, analysis, feature extraction, fusion and compression, among others.

In addition to reducing noise levels in images, denoising techniques must also keep the target associated with the preservation of edges, texture and contrast, which are essential details for image analysis. Towards that end, the bilateral filter (BF) is a spatial filter that brings into account not only the spatial distance between neighboring pixels, but also the difference between their grey intensities [1][2], to calculate the filter weights mask. This structure proves beneficial for reducing noise while preserving thin details.

In the last years, the awareness of the role that BF plays in medical image processing and nondestructive testing has increased [3]. For instance, in [4] it is proposed an iterative $\mathrm{BF}$ for filtering the Rician noise in magnetic resonance images.

Fabiana O. P. Fernandes (fabianapaixaof@gmail.com) is with the Engineering Department, UNA University Center UNA, Brazil.

Flávia M. F. Ferreira (flaviamagfreitas@ pucminas.br) is with the Graduate Program in Electrical Engineering and Electronic and Telecommunication Engineering Department, Pontifical Catholic University of Minas Gerais (PUCMG), Belo Horizonte, Brazil

Zélia M. A. Peixoto (assiszmp@ pucminas.br) is with the Graduate Program in Electrical Engineering and Electronic and Telecommunication Engineering Department, Pontifical Catholic University of Minas Gerais (PUCMG), Belo Horizonte, Brazil.

Andrei S. Silva (andreisantos@ufmg.br) is a graduate student at the Federal University of Minas Gerais (UFMG), Belo Horizonte, Brazil.

Digital Object Identifier: 10.14209/jcis.2018.32
In [5] BF is used to enhance the quality of ultrasound image to determine the fetal health in the early stages of pregnancy and in [6] guided trigonometric BFs are used to improve quality of underwater optical images. Due to its effectiveness in reducing noise while preserving edges, [3] presented a parallel hardware implementation of the BF for real-time applications.

Meanwhile, wavelet-based denoising techniques have been widely applied, usually providing quite reasonable results regarding edge preservation [7]-[14]. Some works have proposed to embed bi-dimensional bilateral filters (2D-BF) in wavelet denoising methods - specifically on the LL sub-band and on the reconstructed image - in order to optimize noise filtering without compromising the edge preservation. This new approach is referred to as multiresolution bilateral filter (MBF) [15][16][17].

In this paper, we present the novel multibandmultiresolution bilateral filter (MMBF), which is derived from the MBF but differs from it by introducing new one-dimensional bilateral filters (1D-BFs) in the sub-bands corresponding to the horizontal and vertical details. Also, an in-field calibration scheme for the embedded 2D-BFs and $1 \mathrm{D}-\mathrm{BF}$ s is also presented to achieve the maximum effectiveness of the MMBF.

Although every kind of image can benefit from bilateral filters and, especially, from MBF and MMBF, as a case study, they were used to process X-ray imagery of phantoms whose densities approximate tissues and arteries of the human body, in an attempt to simulate cerebral and coronary imaging. As the X-ray technology allows the application of fast, accurate and noninvasive procedures, since its inception its use in medical diagnosis has grown in importance when it comes to detect diseases, fractures, among others.

However, during X-ray examinations, more extended procedures require that imagery is carried out under strict conditions of X-ray dosage to avoid long-time exposure of the patient. It obeys the principle "image quality as good as needed", placing a greater emphasis on health safety and less on image quality, as long as it is adequate to enable an accurate diagnosis [18]. As a consequence, the noise level increases, causing a significant reduction in image quality. The noise present in $\mathrm{X}$-ray images can be attributed primarily to the X-ray generator which has a Probability Mass Function (PMF) equivalent to a Poisson random variable [19]. Therefore, the concurrent decrease in the radiation dose and image quality enhancement have been a great concern. Experimental results revealed that the calibrated MMBF boosted the effectiveness of MBF in reducing noise level, concomitantly with the preservation of image edges and details. 
This paper is organized as follows: Section II is dedicated to theoretical background. In Section III, the proposal of the novel MMBF is presented, as well as a complete scheme for calibrating it. In Section IV, we present X-ray phantom images as a case study. Final conclusions are presented in Section V.

\section{THEORETICAL BACKGROUND}

The theoretical background is intended to provide a basis for the filtering process of the MMBF, as well as to characterize quantum noise in X-ray images, presented as a case study.

\section{A. The Bilateral Filter and its Calibration}

The bilateral filter was firstly proposed by [1], consisting of the association of a domain filter and a range filter. This type of filter replaces the intensity of each pixel with a weighted average of intensity values from nearby pixels. For one-dimensional domain, it is given by

$$
I_{f}(k)=\frac{\sum_{n=-N}^{N} W_{d}(n) W_{r}(k, n) I(k-n)}{\sum_{n=-N}^{N} W_{d}(n) W_{r}(k, n)},
$$

in which $I_{f}$ is the filtered image, the weight $W_{d}$ is the spatial domain kernel for smoothing differences in coordinates and $W_{r}$ is the range kernel for smoothing differences in intensities. The original input image is $I, k$ are the coordinates of the current pixel to be filtered, $n$ is the spatial distance between the central pixel and a nearby one, $-N \leq n \leq N$ and the length of the kernel centered in $I(k)$ is $2 N+1$. The normalization term, in the denominator, ensures that the weights for all the pixels add up to one.

A simple and important case of $\mathrm{BF}$ applied to images uses weights based on shift-invariant one-dimensional Gaussian distributions [1], where $W_{d}(n)$ and $W_{r}(k, n)$ depend, respectively, on Euclidean distance of the pixels and on their radiometric distances. They are determined by

$$
\begin{gathered}
W_{d}(n)=\exp \left(\frac{-n^{2}}{2 \sigma_{d^{2}}}\right), \\
W_{r}(k, n)=\exp \left(\frac{-[I(k)-I(k-n)]^{2}}{2 \sigma_{r^{2}}}\right),
\end{gathered}
$$

in which $\sigma_{d}$ and $\sigma_{r}$ are the setting parameters of the decay curves.

As a result, BF replaces a bright pixel at the center of the kernel by an average of the bright pixels in its vicinity and mostly ignores the neighbor dark pixels. Conversely, when the kernel is centered on a dark pixel, the bright pixels under the kernel are ignored instead. Thus, proper filtering behavior is achieved at the boundaries (because of the domain component of the filter), and crisp edges are preserved at the same time (because of the range component).

The calibration of the BF consists in finding optimum values for $\sigma_{d}$ and $\sigma_{r}$, which becomes a cost-benefit analysis for reducing noise and preserving edges, which are competing goals.
In [2], aiming at results regarding image quality, tuning the range filter to the noise level was thought more effective than dealing with the fine tuning of the spatial filter. Based on this argument, a noise-adaptive adjustment of the filter focusing mainly on the parameter $\sigma_{r}$ was introduced and associated with the nonlinear range component. The work suggests that the value $\sigma_{d}$ is calculated so that weights $W_{d}(n)$ in the Gaussian function, for $n= \pm[N+1]$ (that is, out of range of the mask), is forced to less than $10^{-3}$. The goal is making the domain filter similar in performance to an ideal low-pass filter.

Meanwhile, the parameter $\sigma_{r}$ should be calculated concerning the image noise level with $\sigma_{r}=R \sigma_{\text {noise }}$, where $\sigma_{\text {noise }}$ is the standard deviation of noise and $R$ is a factor determined in order to maximize some performance metric, such as Peak Signal to Noise Ratio (PSNR) or Mean Structural Similarity (MSSIM) [21]. The metric MSSIM varies in the range from 0 to 1 (best results reflect on values close to 1) and intends to be close in the effectiveness of human visual system concerning the visual perception. Such approach evaluates quantitatively how the output image approximates a reference image regarding intensity, structure and contrast.

In [2], a database composed by fifty 8-bit images was used. Pictures were corrupted with additive zero-mean Gaussian noise with $\sigma_{\text {noise }}$ varying from 1 to 64 , in steps of 4 ( $\left.\sigma_{\text {noise }}=1: 4: 64\right)$ and the noisy images were processed by $\mathrm{BF}$, setting values of $R$ in the range of 0.5 to 16, in steps of $0.5(R=0.5: 0.5: 16)$. Following, PSNR and MSSIM were calculated separately for each image, for each tuple $\left(\sigma_{\text {noise }}, R\right)$. In the end, the metrics for each tuple were averaged along the images. The characteristic PSNR, referred to as $\mathrm{PSNR}_{c h}$, is given by

$$
\operatorname{PSNR}_{c h}\left(\sigma_{\text {noise }}, R\right)=\frac{\sum_{i=1}^{X} 10 \log \left(\frac{255^{2}}{\operatorname{MSE} x}\right)}{X},
$$

where $\mathrm{PSNR}_{c h}$ is the characteristic PSNR of a set of $X$ images, $\sigma_{\text {noise }}$ is the noise standard deviation and $R$ is the calibration parameter of the range filter. The Mean Square Error between each $x^{\text {th }}$ filtered image $(x=1, \ldots, X)$ and the reference image is $\mathrm{MSE}_{x}$, defined as

$$
\operatorname{MSE}_{x}=\frac{\sum_{q}^{Q} \sum_{p}^{P}\left[I_{f}^{x}(p, q)-I_{r e f}(p, q)\right]^{2}}{P Q},
$$

in which $I_{f}^{x}$ is the $x^{\text {th }}$ filtered image, $I_{r e f}$ is the reference image, $Q$ and $P$ are the number of rows and columns of the image. The characteristic MSSIM, referred to as $\mathrm{MMSIM}_{c h}$, averages the same way as 4 for each tuple $\left(\sigma_{\text {noise }}, R\right)$.

The proposed method for setting $\sigma_{r}$ consisted of searching the value of $R$ that maximizes either $\mathrm{PSNR}_{c h}$ or $\mathrm{MSSIM}_{c h}$ for a specific value of $\sigma_{\text {noise }}$, which is assumed to be known. In order to provide a visual determination of $R$, the values of $\operatorname{PSNR}_{c h}\left(\sigma_{\text {noise }}, R\right)$ were normalized for each specific $\sigma_{\text {noise }}$ using

$$
\operatorname{PSNR}_{n}\left(\sigma_{\text {noise }}, R\right)=\left(1-\frac{P S N R_{\text {ch }}\left(\sigma_{\text {noise }}, R\right)}{\max _{R}\left(P S N R_{\text {ch }}\left(\sigma_{\text {noise }}, R\right)\right)}\right)^{2}
$$


where $\mathrm{PSNR}_{n}$ is the normalized $\mathrm{PSNR}_{c}$ in relation to the maximum value of this metric occuring for $\sigma_{\text {noise }}$. With the proposed normalization, the highest value of $\mathrm{PSNR}_{c h}$ for a specific $\sigma_{\text {noise }}$ is mapped to 0 at $\operatorname{PSNR}_{n}\left(\sigma_{\text {noise }}, R\right)$, while a null value in the first is mapped to 1 in the second.

The optimization procedure proposed in [2] was used to find the value of $R$ for which the normalized metric is null, at each specific $\sigma_{\text {noise }}$. The same normalization procedure can be carried out for the metric MSSIM.

\section{B. Wavelet Denoising and The Multiresolution Bilateral Filter}

Another approach addressed to edge-preservation concomitantly with the reduction of noise level is the wavelet denoising. It consists of three primary stages: (1) decomposition of the image into sub-bands LL (approximation sub-band), LH (horizontal edges sub-band), HL (vertical edges sub-band) and $\mathrm{HH}$ (diagonal details sub-band), (2) thresholding and (3) reconstruction. In the first and third stages, the Discrete Wavelet Transform (DWT) and the Inverse Discrete Wavelet Transform (IDWT), respectively, are performed according to established multiresolution level wavelet basis function. The second stage, in turn, is divided into two steps: (a) calculation of the threshold $\lambda$ and (b) submitting the wavelet coefficients of the noisy image to $\lambda$, aiming to estimate those of the supposedly noise-free image. The calculation of $\lambda$ is what most influences the image quality.

The Neighsrink Stein's Unbiased Risk Estimate (SURE) denoising method [7] is among the most efficient methods of wavelet denoising reported in the literature. It derived from Neighsrink, which assumes the correlation between coefficients to determine the threshold $\lambda$. The neighboring coefficients around a noisy coefficient are used to do an estimate of the noise-free coefficient as

$$
\hat{\theta}_{i, j}=\theta_{i, j}\left(1-\frac{\lambda^{2}}{\sum_{\substack{k=(i-B, \ldots, i, \ldots, i+B) \\ l=(j-B, \ldots, j, \ldots, j+B)}}\left(\theta_{k, l}^{2}\right)}\right) \text {, }
$$

where $\hat{\theta}_{i, j}$ is the estimate of the noise-free coefficient, $\theta_{i, j}$ is the current coefficient to be denoised and $\theta_{k, l}$ are the neighboring coefficients in a square window of dimensions $(2 B+1) \times(2 B+1)$ around $\theta_{i, j}$. The parameter $\lambda$ is the universal threshold, calculated as

$$
\lambda=\sigma_{\text {noise }} \sqrt{2 \log (P)}
$$

where $P$ is the amount of pixels in the image.

In Neighsrink, not only is the size of the window (2B +1 ) invariant throughout the whole process, but also $\lambda$ is kept the same for all sub-bands, which is a substantial disadvantage according to [7]. As a proposal to overcome these limitations, the Neighsrink SURE method [9] aimed at determining optimal values for $\lambda$ and $B$ at each sub-band. The expectation was to minimize the MSE between the noise-free wavelet coefficients and their estimates.
A variant of wavelet denoising is the multiresolution bilateral filter (MBF). It is based on the grounds of [11], which suggests that a two-dimensional bilateral filter (2D-BF) can operate on the LL sub-band aiming at reducing the noise level on the low-frequency coefficients. Instead, the other sub-bands (HL, LH, and $\mathrm{HH}$ ) are subject to universal hard thresholding (UHT). In the end, the reconstructed image is filtered by another 2D-BF. The block diagram of the MBF is depicted in Fig. 1, in which decimation and analysis filters are included in the calculation of DWT, as well as interpolation and synthesis filters are included in the IDWT.

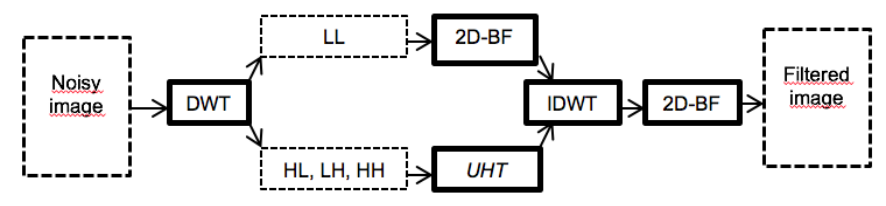

Figure 1. Block Diagram of the MBF.

\section{The Multiband-Multiresolution Bilateral FILTER AND ITS CALIBRATION SCHEME}

\section{A. The Multiband-Multiresolution Bilateral Filter}

Although wavelet-based methods are efficient in image denoising, they are prone to producing artifacts such as lowfrequency noise and edge ringing which relate to the structure of the underlying wavelet [20].

For this reason, this work proposes to investigate the application of one-dimensional bilateral filters (1D-BFs) on the $\mathrm{LH}$ and HL sub-bands, corresponding to horizontal and vertical detail coefficients, instead of subjecting them to UHT, such as in [7][9]. This proposal was based on the assumption that the two-dimensional bilateral filter (2D-BF) performs well when applied to the LL sub-band [11], at which the frequency components are low in both spatial directions. On the same assumption, horizontal and vertical edges must be aligned with the direction in which the spatial frequency components are low. Thus, by hypothesis, it is believed that in the sub-bands LH and HL the application of 1D-BFs toward the horizontal edges (sub-band LH) and vertical edges (sub-band HL) can reduce the noise level, whereas wavelet-based methods would cause low-frequency noise according to [20]. In turn, since the $\mathrm{HH}$ sub-band presents high-frequency components in both directions, it does not apply to bilateral filters. For it, the use of UHT wavelet denoising remains.

Despite the fact that nothing prevents different 1D-BFs from being calibrated for the HL and LH sub-bands, this is usually unnecessary, since noise characterization is directionindependent [19]. Besides that, performing different 1D-BFs would require an additional step in the calibration process to be presented in sub-section III-B, which would be more computationally costly.

Fig. 2 depicts the block diagram of MMBF. It can be faced with Fig. 1 to highlight the differences between the existing $\mathrm{MBF}$ and this novel approach, the MMBF. 


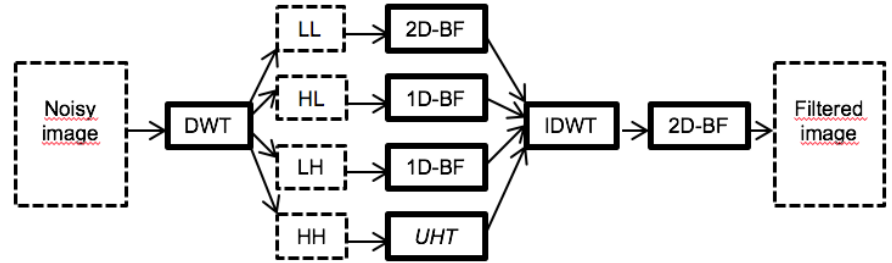

Figure 2. Block Diagram of the novel MMBF

\section{B. Bilateral Filter Calibration: The VAS Scheme}

First, it is proposed an in-field calibration method for the classical BF to be used instead of the one presented in [2]. It is based on a Visual Analysis of Sensitivity to filter's parameters and, for that reason, referred to as VAS Calibration Scheme. It is performed just once for a set of images corrupted by noise with standard deviation $\sigma_{\text {noise }}$. Differently from [2], which does not tune the spatial filter to the noise level, the proposed method is based on the brute-force visual analysis of the sensitivity of a particular performance metric (generally PSNR or MSSIM) to the parameters $\sigma_{d}$ and $R$, making, in the end, $\sigma_{r}=R \sigma_{\text {noise }}$.

The VAS requires that a reference image, presumably noisefree, is available for the calculation of $\sigma_{\text {noisy }}$ as well as of PSNR or MSSIM (in fact, any other measuring can be used). The average metric is evaluated both for the noisy (e.g., $\mathrm{PSNR}_{\text {noisy }}$ ) and the filtered images (e.g., $\mathrm{PSNR}_{c h}$ ).

The VAS method is implemented in the following steps, exemplified in the case of using the PSNR as the chosen metric.

1) Normalize the pixels of the reference and noisy images from 0 to 1 to make the calibration procedure independent from bit-depth. This has not been identified as a concern in [2].

2) Estimate $\sigma_{\text {noise }}$ from the average error histogram between the noisy frames and the reference image.

3) Calculate PSNR ${ }_{\text {noisy }}$, the average PSNR for the noisy images relatively to the reference image.

4) Vary $\sigma_{d}$ and $R$ at 200 different tuples $\left(\sigma_{d}, R\right)$, with $\sigma_{d}=0.5: 0.5: 5$ and $R=0.5: 0.5: 10$ and for each tuple calculate $\operatorname{PSNR}_{c h}\left(\sigma_{d}, R\right)$ as in (4) for the images filtered with BF.

5) Calculate and plot the gain obtained from the use of BF, referred to as $G\left(\sigma_{d}, R\right)$, according to (9). From this plot, choose a $\sigma_{d}$ that results in the largest gains.

6) Calculate and plot the normalized metric $\mathrm{PSNR}_{n}$ as in (6) to visualize the optimum $R$ for a chosen $\sigma_{d}$.

$$
G\left(\sigma_{d}, R\right)=P S N R_{c h}\left(\sigma_{d}, R\right)-P S N R_{\text {noisy }}(d B)
$$

\section{The VAS Calibration Scheme for the MMBF}

This work presents a proposal to adjust the parameters of the MMBF so that each embedded bilateral filter (two 2D-BFs and two 1D-BFs) is calibrated using the VAS method. After choosing a specific metric (usually, PSNR or MSSIM), the complete calibration of the MMBF is accomplished in four steps described in the following, taking the metric PSNR as an example.

1) Estimate $\sigma_{\text {noise }}$ and PSNR $\mathrm{Pnoisy}_{\text {. }}$

2) Calibrate the first 2D-BF (applied to LL sub-band) using VAS, by performing the secondary stages:

a) Perform the DWT to obtain LL, HL, LH and HH sub-bands;

b) For every tuple $\left(\sigma_{d}, R\right)$, do:

i) Use a $2 \mathrm{D}-\mathrm{BF}$ to operate the LL coefficients;

ii) Synthesize the denoised image with the IDWT;

iii) Calculate $\mathrm{PSNR}_{c h}$ of the filtered images;

iv) Calculate and plot $G\left(\sigma_{d}, R\right)$ in order to choose the $\sigma_{d}$ that results in the largest gains of the 2D-BF;

v) Calculate and plot $\mathrm{PSNR}_{n}$ in order to visualize the optimum $R$ for the chosen $\sigma_{d}$.

c) Keep the chosen tuple $\left(\sigma_{d}, R\right)$ for the $2 \mathrm{D}-\mathrm{BF}$ applied to LL sub-band unchanged in steps 3 and 4 .

3) Calibrate the two equal 1D-BFs (applied to both HL and LH sub-bands) using VAS, by performing the secondary stages:

a) Perform the DWT to obtain LL, HL, LH and HH sub-bands;

b) Process LL coefficients with the 2D-BF calibrated in step 2;

c) For every tuple $\left(\sigma_{d}, R\right)$, do:

i) Use the $1 \mathrm{D}-\mathrm{BFs}$ to operate both $\mathrm{HL}$ and $\mathrm{LH}$ coefficients;

ii) Synthesize the processed image with the IDWT;

iii) Calculate $\mathrm{PSNR}_{c h}$ of the filtered images;

iv) Calculate and plot $G\left(\sigma_{d}, R\right)$ in order to choose the $\sigma_{d}$ that results in the largest gains of the 1D-BFs.

v) Calculate and plot $\mathrm{PSNR}_{n}$ in order to visualize the optimum $R$ for the chosen $\sigma_{d}$.

vi) Keep the chosen tuple $\left(\sigma_{d}, R\right)$ for the two 1D-BFs unchanged in step 4.

4) Calibrate the second 2D-BF (applied to the reconstructed image) using VAS, by performing the secondary stages:

a) Perform the DWT to obtain LL, HL, LH and HH sub-bands;

b) Process LL coefficients with the 2D-BF calibrated in step 2;

c) Process HL and LH coefficients with the 1D-BFs calibrated in step 3;

d) Treat the HH coefficients by the UHT method;

e) Synthesize the denoised image with the IDWT;

f) For every tuple $\left(\sigma_{d}, R\right)$, do:

i) Use the $2 \mathrm{D}-\mathrm{BF}$ to operate the synthesized image;

ii) Calculate $\mathrm{PSNR}_{c h}$ of the filtered images;

iii) Calculate and plot $G\left(\sigma_{d}, R\right)$ to choose the $\sigma_{d}$ that results in the highest gains of the 2D-BF applied to the reconstructed image;

iv) Calculate and plot $\mathrm{PSNR}_{n}$ to visualize the optimum $R$ for this second 2D-BF. 
Finally, the tuples $\left(\sigma_{d}, R\right)$ obtained at steps 2,3 and 4 are the setting parameters of the MMBF.

\section{CASe Study: MMBF ApPlied to X-Ray IMAges}

Since it is always interesting to count on image quality regarding sharpness, contrast, noise reduction and lower delay in the preview, the fluoroscopic images described in Section I must be filtered by assuring the preservation of their edge profile [18]. According to [22], it is of paramount importance that any methodology used to improve quality and safety takes into account the dynamic nature and multiplicity of factors affecting them. However, as far as we know, the literature lacks procedures to perform in-field calibrations of the systems used to enhance quality of low-dose X-ray images, which are corrupted with Poisson noise [19]. These images are affected by individual attributes, technology, clinical context, and exam type, according to [22][23].

The subsection IV-A presents the VAS calibrating approach presented in III-B to tune a BF applied to noisy X-ray phantom images, shown as a case study. Following, IV-B presents the parameters resulting from the VAS calibration of the BFs embedded in MBF and MMBF. At last, IV-C presents the quantitative and qualitative assessment of $\mathrm{MBF}$ and $\mathrm{MMBF}$ applied to the X-ray image of a testing phantom.

The MATLAB files for calibration and filtering and the phantom images used in the experiments can be accessed in [24].

\section{A. The VAS Calibration of BF applied to X-ray images}

The VAS calibration method for BF applied to images of a phantom acquired with actual X-ray doses in fluoroscopy leads to $\sigma_{\text {noise }}=0.027$. The phantom presents density characteristics that simulate different tissues of the human body, such as blood vessels, bones and calcifications, as well as other standard structures present in medical procedures, such as catheters and prostheses. Fig. 3 depicts the calibration phantom image generated with the equipment AngiX III FD - GE Healthcare.

The reference image necessary to accomplish the VAS calibration can be obtained with high doses of X-ray. However, if it is not practical, the reference image $I_{r e f}$ can be estimated by averaging $M$ consecutive $\mathrm{X}$-ray noisy images of a calibration phantom. The calibration is performed just once for a specific X-ray dosage, which determines $\sigma_{\text {noise }}$. Then, all the medical procedures undertaken at equivalent doses can be held without any updates of $\sigma_{d}$ and $R$.

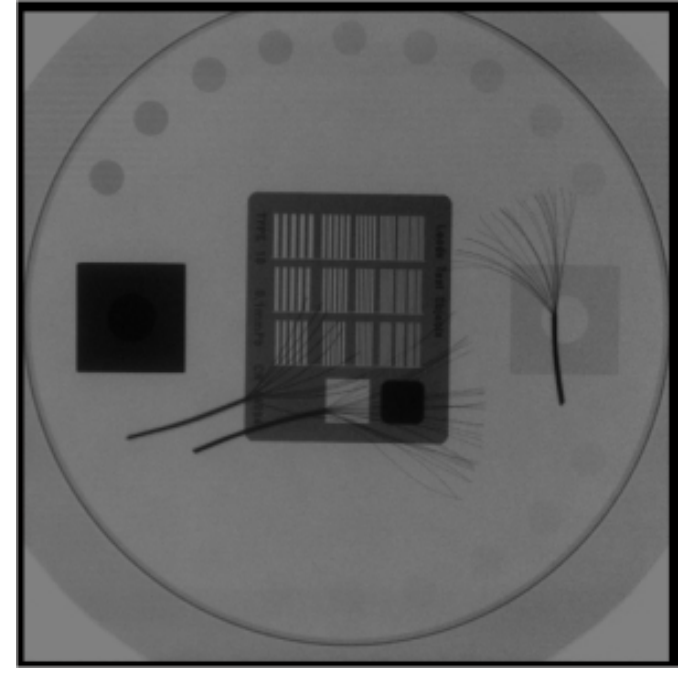

Figure 3. A noisy image of the calibration phantom.

Graphs shown in Figures 4 and 5 assume the chosen metric is either PSNR (sub-figures a) or MSSIM (sub-figures b). Fig. 4 shows a visual analysis of the gain $G\left(\sigma_{d}, R\right)$ obtained by $\mathrm{BF}$, showing that the best performance of the filter is obtained with $\sigma_{d}$ greater than 2 . In this case study, this parameter was set to 3 .

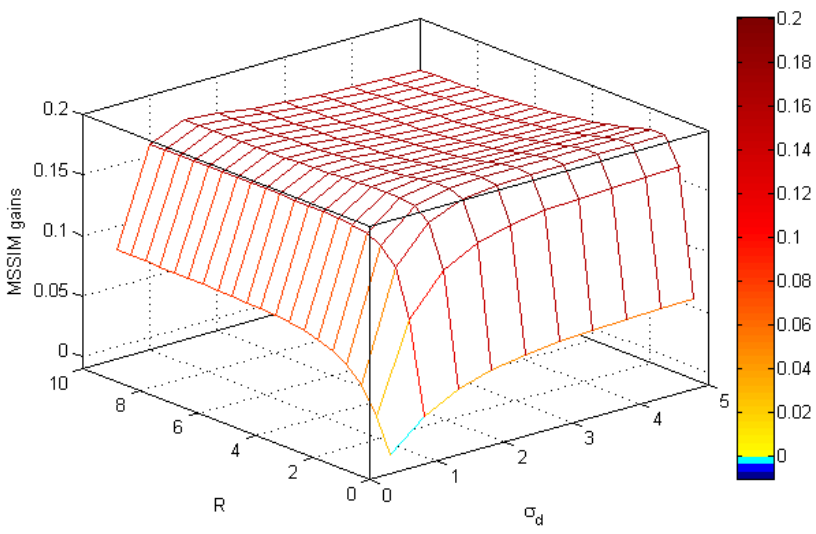

(a)

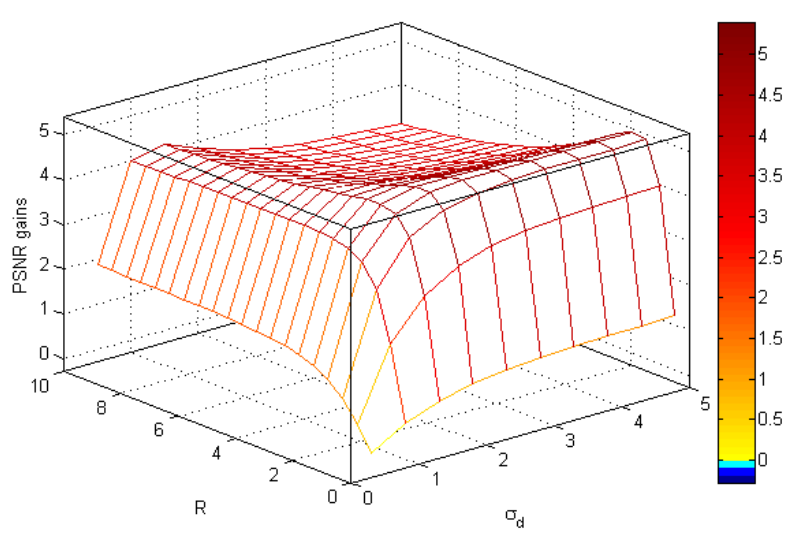

(b)

Figure 4. $G\left(\sigma_{d}, R\right)$ obtained by BF when processing calibration phantom: (a) Gains of $\operatorname{PSNR}\left(\sigma_{d}, R\right)(\mathrm{dB})$; (b) Gains of $\operatorname{MSSIM}\left(\sigma_{d}, R\right)$ 
Fig. 5 shows the normalized metrics obtained from the calibration phantom. They aim to demonstrate the value of $R$ that optimizes the filter performance for a given amount of $\sigma_{d}$, as it is assigned a zero value in the z-axis.

It can be verified in Fig. 5 that the lower $\sigma_{d}$, the larger the expected value of $R$ in order to optimize performance. However, for values of $\sigma_{d}$ greater than 2.5 , the optimum $R$ keeps invariant and equal to 2 .

It is worth noticing that a typical box filter would be equivalent to $\mathrm{BF}$ with the highest values of $\sigma_{d}$ and $R$, since all the neighbor pixels inside the mask have to be weighed with the same amount, no matter their distances from the central pixel of the mask or their intensities. As it can be visualized in Fig. 5a and Fig. 5b, the box filter corresponds to the worst performance of BF.

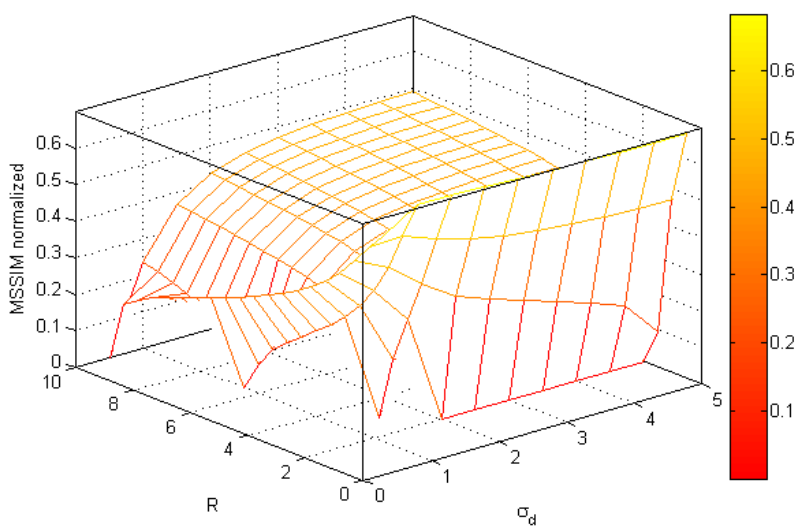

(a)

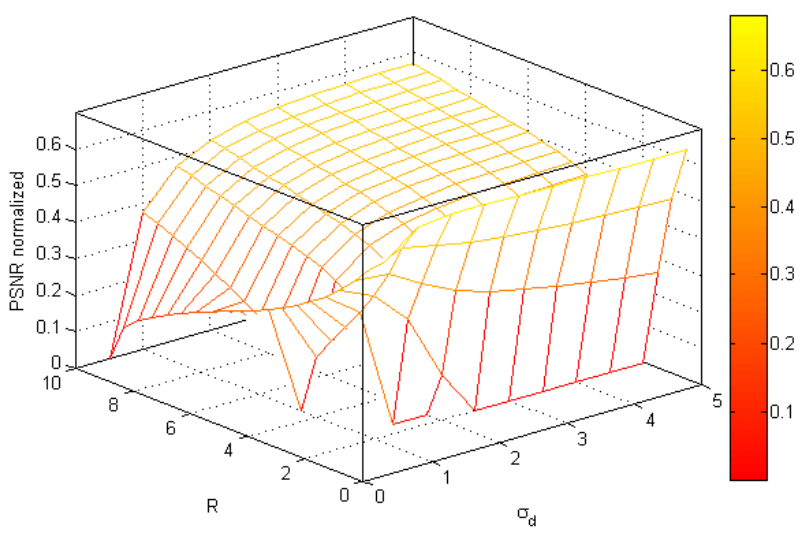

(b)

Figure 5. Normalized metrics at the calibration phantom: (a) $P S N R_{n}\left(\sigma_{d}, R\right)$ (dB); (b) $M S S I M_{b}\left(\sigma_{d}, R\right)$

It is concluded from the calibration based on VAS that reasonable values for the setting parameters of $\mathrm{BF}$ when $\sigma_{\text {noise }}$ is 0.027 can be $\sigma_{d}=3$ and $R=2$, using a $7 \times 1$ mask. These values can optimize both PSNR and MSSIM.

\section{B. The VAS Calibration of MMBF Applied to X-ray Images}

In the following, the complete calibration scheme for the MMBF presented in subsection III-C was performed using the same calibration phantom. The final values for $\sigma_{d}$ and $R$ are shown in Table I for five different noise standard deviations. The first one, $\sigma_{\text {noise }}=0.027$, approximates the noise level in images acquired with actual X-ray dose in fluoroscopy. The larger ones were added for the sole purpose of analysing the behaviour of the filter performance. The values of $\sigma_{d}$ were chosen using either $\mathrm{PSNR}_{c h}$ or $\mathrm{MSSIM}_{c h}$ to calculate $G\left(\sigma_{d}, R\right)$. The values of $R$, in turn, were determined from the normalized graphs of the chosen metric $\left(\mathrm{PSNR}_{n}\right.$ or $\left.\mathrm{MSSIM}_{n}\right)$ assuming the chosen $\sigma_{d}$.

Table I

$\sigma_{d}$ AND $R$ VALUES AT DIFFERENT NOISE LEVELS FOR MMBF

\begin{tabular}{|c|c|c|}
\hline Parameters & $\sigma_{d}$ & $\mathrm{R}$ \\
\hline \multicolumn{3}{|l|}{$\overline{\sigma_{\text {noise }}}=0.027$} \\
\hline 2D-BF applied to LL & 3 & 2.5 \\
\hline 1D-BF applied to the LH and HL & 3 & 3 \\
\hline 2D-BF applied to reconstructed images & 3 & 0.5 \\
\hline \multicolumn{3}{|l|}{$\overline{\sigma_{\text {noise }}}=0.045$} \\
\hline 2D-BF applied to LL & 3 & 2 \\
\hline 1D-BF applied to the LH and HL & 3 & 8.5 \\
\hline 2D-BF applied to reconstructed images & 3 & 0.5 \\
\hline \multicolumn{3}{|l|}{$\overline{\sigma_{\text {noise }}}=0.077$} \\
\hline 2D-BF applied to LL & 3 & 2.5 \\
\hline 1D-BF applied to the LH and HL & 5 & 15 \\
\hline 2D-BF applied to reconstructed images & 3 & 1 \\
\hline \multicolumn{3}{|l|}{$\overline{\sigma_{\text {noise }}}=0.092$} \\
\hline 2D-BF applied to LL & 5 & 2 \\
\hline 1D-BF applied to the $\mathrm{LH}$ and HL & 7.5 & 13 \\
\hline 2D-BF applied to reconstructed images & 2.5 & 1 \\
\hline \multicolumn{3}{|l|}{$\overline{\sigma_{\text {noise }}}=0.122$} \\
\hline 2D-BF applied to LL & 5 & 2 \\
\hline 1D-BF applied to the $\mathrm{LH}$ and HL & 5.5 & 22.5 \\
\hline 2D-BF applied to reconstructed images & 2 & 1.5 \\
\hline
\end{tabular}

The greater the noise, the smaller is the difference between the $R$-values of the two bilateral filters (2D-BFs) applied at different resolution levels (LL sub-band against reconstructed image). Comparing different sub-bands at the same level (LL against LH and HL sub-bands), the $R$-values are closer for a smaller noise content $\left(\sigma_{\text {noise }}=0.027\right)$, but their difference increases as the noise reaches greater values. As more evident the noise becomes, more the $R$-value in the one-dimensional bilateral filters (1D-BFs) increases. It means that a wider range of intensity have to be used at the range filter, according to (2), in an attempt to find more pixels in the neighborhood aiming to attenuate the noise and calculate the pixel value of the processed image. This will obviously have a negative impact on the preservation of image edges.

\section{Filtering $X$-Ray Image with $M B F$ and $M M B F$}

With the purpose of comparing the two approaches, MBF and MMBF, both were calibrated using the VAS method. The calibration of the 2D-BF applied to the approximation coefficients (LL sub-band) yields values for $\sigma_{d}$ and $R$ which are the same for either MMBF or MBF. The calibration of the second $2 \mathrm{D}-\mathrm{BF}$, applied to the reconstructed images, led to different values for the parameters in MMBF and MBF, for $\sigma_{\text {noise }}$ equal or higher than 0.077 . The parameters found for this filter in MBF are listed in Table II. 
Table II

$\sigma_{d}$ AND $R$ VALUES AT DIFFERENT NOISE LEVELS FOR THE $2 \mathrm{D}-\mathrm{BF}$ APPLIED TO THE RECONSTRUCTED IMAGES AT MBF

\begin{tabular}{lcc}
\hline \hline$\sigma_{\text {noise }}$ & $\sigma_{d}$ & $\mathrm{R}$ \\
\hline$\overline{0.027}$ & 3 & 0.5 \\
\hline$\overline{0.045}$ & 3 & 0.5 \\
\hline$\overline{0.077}$ & 3 & 0.5 \\
\hline \hline 0.092 & 1.5 & 0.5 \\
\hline \hline 0.122 & 1.5 & 0.5 \\
\hline \hline
\end{tabular}

Then, they were used to reduce the noise level at X-ray images of a test phantom acquired by AngiX III FD. The PSNR and MSSIM values of a noisy image of the testing phantom and its corresponding reconstructed image after being filtered with MBF or MMBF are presented on Table III for five different values of $\sigma_{\text {noise }}$, corresponding to the usual X-ray dose in fluoroscopy $\left(\sigma_{\text {noise }}=0.027\right)$ and four larger ones, with the sole purpose of analysing the behaviour of the filter performance. The best values of PSNR and MSSIM obtained are highlighted in bold.

For larger values of $\sigma_{\text {noise }}$, the gains of the MMBF decrease, becoming smaller than the gains of the MBF for images with higher noise level. But with $\sigma_{\text {noise }}$ equal to 0.027 , the focus of our analysis, MMBF provided gains of approximately $1.2 \mathrm{~dB}$ in PSNR when compared to MBF, while the increment in MSSIM was $7 \%$, rising from 0.84 to 0.90 .

Table III

PSNR AND MSSIM USING AN IMAGE FROM THE TEST PHANTOM

\begin{tabular}{lcc}
\hline \hline & & \\
Filter & PSNR $(\mathrm{dB})$ & MSSIM \\
\hline \hline$\sigma_{\text {noise }}=0.027$ & & \\
\hline (no filter) & 30.97 & 0.75 \\
MBF & 33.83 & 0.84 \\
MMBF & $\mathbf{3 5 . 2 2}$ & $\mathbf{0 . 9 0}$ \\
\hline \hline$\sigma_{\text {noise }}=0.045$ & & \\
\hline (no filter) & 26.69 & 0.52 \\
MBF & 32.60 & 0.80 \\
MMBF & $\mathbf{3 3 . 4 9}$ & $\mathbf{0 . 8 5}$ \\
\hline \hline$\sigma_{\text {noise }}=0.077$ & & 0.29 \\
\hline (no filter) & 22.10 & $\mathbf{0 . 6 9}$ \\
MBF & $\mathbf{3 0 . 1 8}$ & $\mathbf{0 . 6 9}$ \\
MMBF & 30.06 & 0.23 \\
\hline \hline$\sigma_{\text {noise }}=0.092$ & & $\mathbf{0 . 7 1}$ \\
\hline (no filter) & 20.48 & 0.66 \\
MBF & $\mathbf{3 0 . 0 1}$ & 0.16 \\
MMBF & 29.36 & $\mathbf{0 . 6 7}$ \\
\hline \hline$\sigma_{\text {noise }}=0.122$ & & 0.65 \\
\hline (no filter) & 18.17 & $\mathbf{2 9 . 0 7}$ \\
MBF & 28.40 & \\
MMBF & & \\
\hline \hline
\end{tabular}

A subjective analysis of the results provided by MBF and MMBF can be performed from inspection. Figs. 6a, $6 \mathrm{~b}$ and $6 \mathrm{c}$ (respectively, the noisy image and the images processed by MBF and MMBF) show results for $\sigma_{\text {noise }}$ is 0.027 . The three images had their edges highlighted by the same contrastequalization algorithm, for better analysis. It can be verified that $\mathrm{MMBF}$ is elected as the best filtering option since it achieves the best cost-benefit regarding preserving the edges while reducing the noise level. As a starting point, the reader should zoom the regions indicated with arrows in Fig. $6 \mathrm{~b}$ and 6c. It is quite clear that in the second, the stripes have been preserved, while in the first there is a haze across the region.

It is crucial to highlight that the VAS in-field calibration method can be accomplished with any metrics that are better suited to the user's expectations, and it is not restricted to PSNR or MSSIM. In this context, the specialist can also apply his own subjective evaluation or a Quality-Safety Index [2] to determine the tuple of parameter values that optimize performance.

\section{CONCLUSIONS}

Recent publications have presented the MBF as a means of improving the capability of reducing noise and preserving image details, usually applying wavelet techniques to the lowfrequency coefficients as well as to the reconstructed image. Here, we introduced the MMBF, a novel approach to the $\mathrm{MBF}$, as a contribution regarding the use of bilateral filters in multiple sub-bands of the same multiresolution level of wavelet domain.

After investigating several proposals concerning the $\mathrm{BF}$ and MBF structures, it could be observed a lack of efficient procedures to calibrate the parameters of this type of filter when applied to images of any bit-depth. We also presented, in this report, a new and accurate in-field calibration procedure for the bilateral filters based on a visual analysis of sensitivity to its setting parameters (VAS), through the use of images of a calibration phantom. In-field calibration consists of a useful and practical tool concerning the nature and multiplicity of factors which affect the quality of dynamic images.

We have presented a case study based on X-ray phantoms and used the VAS method to calibrate the bilateral filters embedded into both $\mathrm{MBF}$ and MMBF. It was possible to conclude that for high noise levels the use of one-dimensional bilateral filters in the HL and LH sub-bands (MMBF) is no longer relevant if compared to UHT use (MBF). Under such circumstances, MMBF achieved similar or lower level of performance than MBF. However, for noise levels compatible with usual X-ray doses in fluoroscopy, it was demonstrated the best performance of the MMBF on preserving image details while reducing noise, surpassing the capacity of the MBF. It was verified not only from objective metrics calculated for test phantom images, such as PSNR and MSSIM, but also through visual inspection of one of the frames. In general, results indicate a promising future for the $\mathrm{MMBF}$ in image processing applications.

\section{REFERENCES}

[1] C. Tomasi and R. Manduchi, "Bilateral filtering for gray and color images", in Sixth International Conference on Computer Vision, Bombay, India, pp. 839-846, 1998, doi: 10.1109/ICCV.1998.710815.

[2] A. Gabiger, M. Kube, P. Schmitt, R. Weigel and R. Rose, "Image denoising using bilateral filter with noise-adaptive parameter tuning", in 37th Annual Conference on IEEE Industrial Electronics Society, Melbourne, VIC, Australia, pp.4515-4520, 2011, doi: 10.1109/IECON.2011.6120053.

[3] A. Gabiger-Rose, M. Kube, R. Weigel and R. Rose, "An FPGA-Based fully synchronized design of a bilateral filter for real-time image denoising", IEEE Transactions on Industrial Electronics, Vol. 61, Issue 8, pp. 4093 - 4104, 2014, doi: 10.1109/TIE.2013.2284133. 


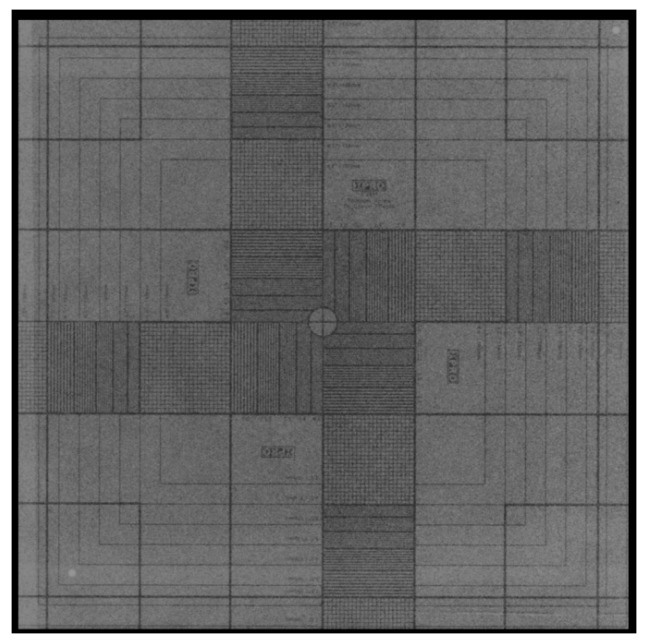

(a)

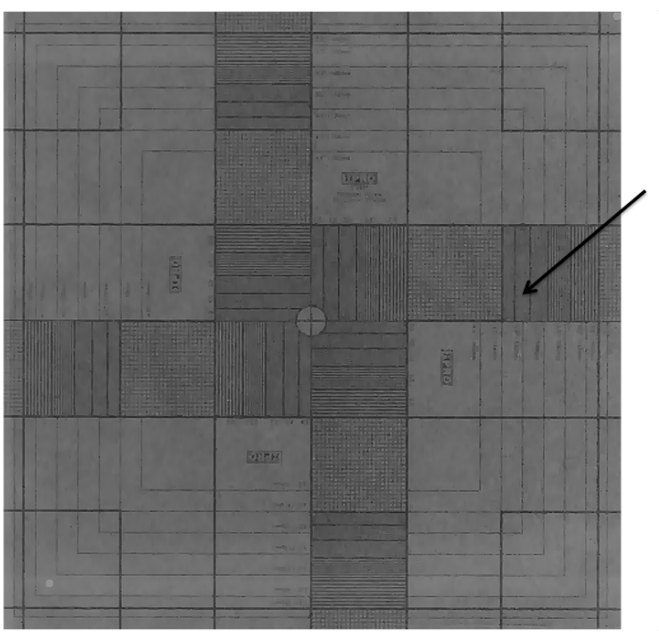

(b)

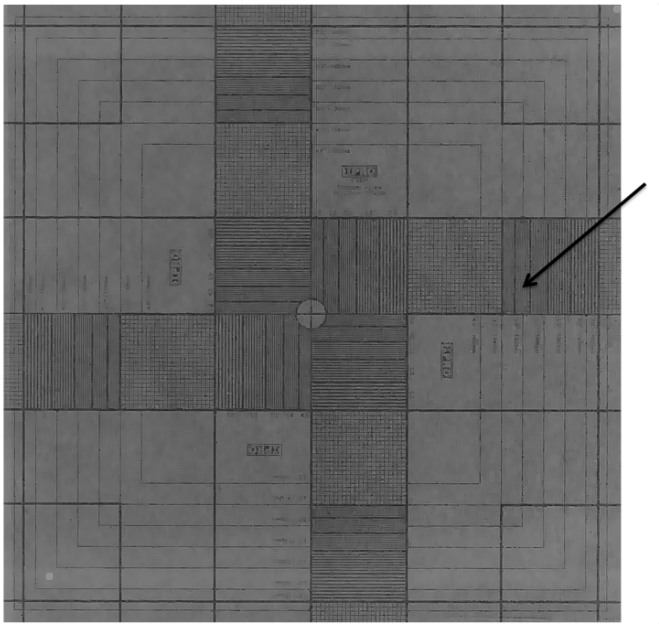

(c)

Figure 6. Phantom test images for $\sigma_{\text {noise }}=0.027$ : (a) Noisy Image $(\mathrm{PSNR}=30.97 \mathrm{~dB}$ and $\mathrm{MSSIM}=0.75)$; (b) Image filtered with MBF $(\mathrm{PSNR}=33.83 \mathrm{~dB}$ and $\mathrm{MSSIM}=0.84)$; (c) Image filtered with MMBF $(\mathrm{PSNR}=35.22 \mathrm{~dB}$ and $\mathrm{MSSIM}=0.90)$
[4] R. Riji, J. Rajan, J. Sijbers an M. S. Nair, "Iterative bilateral filter for Rician noise reduction in MR images", Signal, Image and Video Processing SIViP, Vol. 9, Issue 7, pp. 1543-1548, 2015, doi:10.1007/s11760-0130611-6.

[5] G. A. Almira, T. Harsono, R. Sigit, I. G. N. T. B. Bimantara and J. S. M. Saputra, "Performance analysis of gaussian and bilateral filter in case of determination the fetal length", in International Conference on Knowledge Creation and Intelligent Computing, Manado, Indonesia, pp. 246 - 252, 2016, doi: 10.1109/KCIC.2016.7883654.

[6] H. Lu, Y. Li and S. Serikawa, "Underwater image enhancement using guided trigonometric bilateral filter and fast automatic color correction", in 20th IEEE International Conference on Image Processing, Melbourne, VIC, Australia, 2013, doi: 10.1109/ICIP.2013.6738704.

[7] G. Y. Chen, T. D. Bui and A. Krzyzak, "Image denoising using neighbouring wavelets coefficients", in IEEE International Conference on Acoustics, Speech and Signal Processing, Montreal, Canada, pp. 917-920, 2004, doi: 10.1109/ICASSP.2004.1326408.

[8] L. Wang, J. Lu and Y. Li, "Noise removal for medical X-ray images in multiwavelet domain", International Journal of Image and Graphics, Vol. 08, Issue 01, pp. 594-597, 2008, doi: 10.1142/S0219467808002952.

[9] Z. Dengwen and C. Wengang, "Image denoising with an optimal threshold and neighbouring window", Pattern Recognition Letters, Vol. 29, Issue 11, pp. 1694-1697, 2008, doi:10.1016/j.patrec.2008.04.014

[10] M. Sakata and K. Ogawa, "Noise reduction and contrast enhancement for small-dose X-ray images in wavelet domain", in. IEEE Nuclear Science Symposium Conference Record, Orlando, USA, pp. 2924-2929, 2009, doi: 10.1109/NSSMIC.2009.5401610.

[11] V. N. P. Raj and T. Venkateswarlu, "Denoising of medical images using undecimated wavelet transform", in IEEE Recent Advances in Intelligent Computational Systems, pp. 483-488, Trivandrum, India, 2011, doi: 10.1109/RAICS.2011.6069359.

[12] I. Tache, A. Udrea, D. Stefanoiu and C. Lupu, "Enhanced visualization of cerebral blood vessels for X-ray angiograms", in 4th IEEE International Conference on E-Health and Bioengineering, pp. 1-4, Iasi, Romania, 2013, doi: 10.1109/EHB.2013.6707406.

[13] P. Hill, A. Achim, M. E. Al-Mualla and D.Bull, "Contrast sensitivity of the wavelet, dual-tree complex wavelet, curvelet and steerable pyramid transforms", IEEE Transactions on Image Processing, vol. 25, no. 6, pp. 2739-2751, 2016, doi: 10.1109/TIP.2016.2552725.

[14] S. Lahmiri, "Denoising techniques in adaptive multi-resolution domains with applications to biomedical images", Healthcare Technology Letters, vol. 4, no. 1, pp. 25-29, 2016, doi:10.1049/htl.2016.0021.

[15] M. Zhang and B. K. Gunturk, "Multiresolution bilateral filtering for image denoising", IEEE Transactions on Image Processing, vol. 17, no.12, pp. 2324-2333, 2008, doi:10.1109/TIP.2008.2006658.

[16] N. Rajpoot and I. Butt, "A multiresolution framework for local similarity based image denoising", Pattern Recognition Letters, vol. 45, pp. 2938-2951, 2012, doi:10.1016/j.patcog.2012.01.023

[17] B. Jin, S. J. You and N. I. Cho, "Bilateral image denoising in the Laplacian Subbands", EURASIP Journal on Video and Processing, vol. 26, pp. 1-12, 2015, doi: 10.1186/s13640-015-0082-5.

[18] E. Vano, "ICRP recommendations on managing patient dose in digital radiography", Radiation Protection Dosimetry, Vol. 114, Issue 1-3, pp 126-130, 2005, doi:10.1093/rpd/nch533

[19] J. L. Prince and J. M. Links. Medical imaging signals and systems. Prentice-Hall, 2005.

[20] H. Yu, L. Zhao and H. Wang, "Image Denoising Using Trivariate Shrinkage Filter in the Wavelet Domain and Joint Bilateral Filter in the Spatial Domain", IEEE TRANSACTIONS ON IMAGE PROCESSING, Vol. 18, Number 10, pp 2364-2369, 2009, doi: 10.1109/TIP.2009.2026685.

[21] Z. Wang, A. C. Bovik, H. R. Sheikh and E.P. Simoncelli, "Image quality assessment: From error visibility to structural similarity", IEEE Transactions on Image Processing, vol. 13, Issue 4, pp. 600-612, 2004, doi: 10.1109/TIP.2003.819861.

[22] B. I. Reidner, "The quality/safety medical index: a standardized method for concurrent optimization of radiation dose and image quality",Journal of Digital Imaging, vol. 28, Issue 1, pp. 1-6, 2015, doi: 10.1007/s10278014-9747-3.

[23] L. Zhang, J. Chen, Y. Zhu and Jianhua Luo, "Comparisons of several new denoising methods for medical images", in 3rd Int. Conf. Bioinformatics and Biomedical Engineering, Beijing, India, pp. 1-4, 2009, doi: 10.1109/ICBBE.2009.5162543.

[24] Gradute Program of Electrical Enguneering of Pontifical Catholic University of Minas Gerais, "Access to Matlab Files and Phantom Images", https://www.dropbox.com/sh/buajm01gnred1hz/AAAsus1AxrNi4OxPbTnVRsta?dl=0/. 


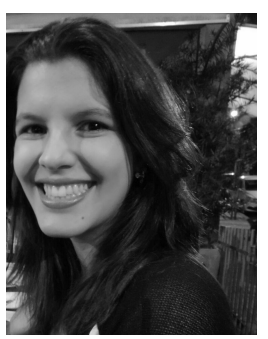

Fabiana Oliveira Paixão Fernandes received her bachelor degree in Electronic and Telecommunication Engineering in 2010 and her Master degree in Electrical Engineering in 2013, both by the Pontifical Catholic University of Minas Gerais (PUC Minas), having worked with image processing. She is currently an undergraduate professor at University Center (UNA) working on signal and systems.

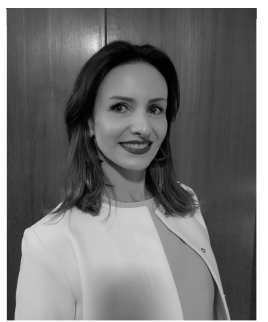

Flávia Magalhães Freitas Ferreira graduated in Electrical Engineering at Pontifical Catholic University of Minas Gerais (PUC Minas) in 1990, concluded her Master degree at the Federal University of Minas Gerais in 1995 and her Ph.D. at Pontifical Catholic University of Rio de Janeiro in 2004. She is a Professor at the Graduate Program in Electrical Engineering and the Department of Electronic and Telecommunication Engineering at PUC Minas, working on signal and image processing and computing vision.

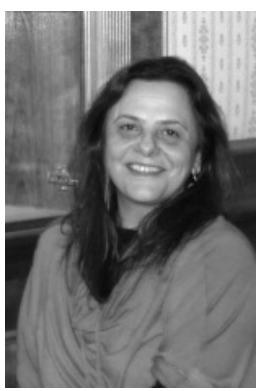

Zelia Myriam Assis Peixoto graduated in Electronic and Telecommunication Engineering at Pontifical Catholic University of Minas Gerais (PUC Minas) in 1981 and received her Master degree and Ph.D. degree from the Federal University of Minas Gerais in 1995 and 2000, respectively. She is a Professor in the Graduate Program in Electrical Engineering and at the Department of Electronic and Telecommunication Engineering at PUC Minas, with main research topics including digital signal processing and system identification.

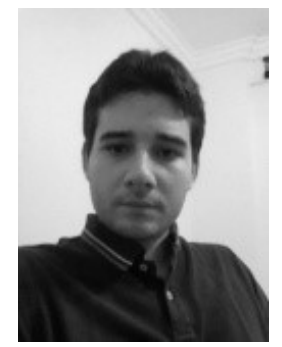

Andrei Santos Silva Andrei S. Silva received his bachelor degree in Electronic and Telecommunication Engineering from the Pontifical Catholic University of Minas Gerais in 2015, having worked in his undergraduate thesis with image processing and object tracking. His Master degree was received from the Federal University of Minas Gerais in 2017, having worked with integrated circuits reliability and Electronic Design Automation (EDA). 